\title{
BMJ Open Association of early adult modifiable cardiovascular risk factors with left atrial size over a 20-year follow-up period: the CARDIA study
}

\author{
Anderson C Armstrong, ${ }^{1,2}$ Samuel S Gidding, ${ }^{3}$ Laura A Colangelo, ${ }^{4}$ Satoru Kishi, ${ }^{1}$ \\ Kiang Liu, ${ }^{4}$ Stephen Sidney, ${ }^{5}$ Suma Konety, ${ }^{6}$ Cora E Lewis, ${ }^{7}$ Luís C L Correia, ${ }^{8}$ \\ Joao A C Lima ${ }^{1}$
}

To cite: Armstrong AC, Gidding SS, Colangelo LA, et al. Association of early adult modifiable cardiovascular risk factors with left atrial size over a 20year follow-up period: the CARDIA study. BMJ Open 2014;4:e004001. doi:10.1136/bmjopen-2013004001

- Prepublication history and additional material for this paper is available online. To view these files please visit the journal online (http://dx.doi.org/10.1136/ bmjopen-2013-004001).

Received 12 September 2013 Revised 21 November 2013 Accepted 22 November 2013

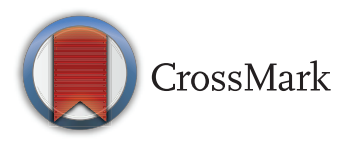

For numbered affiliations see end of article.

Correspondence to Dr Joao AC Lima; jlima@jhmi.edu

\section{ABSTRACT}

Objectives: We investigate how early adult and 20-year changes in modifiable cardiovascular risk factors (MRF) predict left atrial dimension (LAD) at age 43-55 years.

Methods: The Coronary Artery Risk Development in Young Adults (CARDIA) study enrolled black and white adults (1985-1986). We included 2903 participants with echocardiography and MRF assessment in followup years 5 and 25. At years 5 and 25, LAD was assessed by M-mode echocardiography, then indexed to body surface area (BSA) or height. Blood pressure (BP), body mass index (BMI), heart rate (HR), smoking, alcohol use, diabetes and physical activity were defined as MRF. Associations of MRF with LAD were assessed using multivariable regression adjusted for age, ethnicity, gender and year-5 left atrial (LA) size.

Results: The participants were $30 \pm 4$ years; $55 \%$ white; $44 \%$ men. $L A D$ and $L A D /$ height were modest but significantly higher over the follow-up period, but $L A D$ / BSA decreased slightly. Increased baseline and 20-year changes in BP were related to enlargement of $L A D$ and indices. Higher baseline and changes in BMI were also related to higher $L A D$ and $L A D /$ height, but the opposite direction was found for LAD/BSA. Increase in baseline $H R$ was related to lower $L A D$ but not $L A D$ indices, when only baseline covariates were included in the model. However, baseline and 20-year changes in HR were significantly associated to LA size.

Conclusions: In a biracial cohort of young adults, the most robust predictors for LA enlargement over a 20-year follow-up period were higher BP and BMI. However, an inverse direction was found for the relationship between BMI and LAD/BSA. HR showed an inverse relation to LA size.

\section{INTRODUCTION}

Left atrial (LA) remodelling is an important independent predictor of cardiovascular (CV) events in diverse populations. LA is structurally and functionally linked to the left ventricle, functioning as a reservoir during

\section{Strengths and limitations of this study}

We show the long-term effect of modifiable cardiovascular risk factors on left atrial size, over a 20-year follow-up period.

- This large cohort study helps understanding the role of risk factors on the left ventricular filling pressures over young adulthood.

- We used the left atrial diameter assessment by M-mode echocardiography, a practical, low cost and validated method. However, it may lack accuracy as it is based on the linear measurement of the anteriorposterior diameter and may not account for the left atrial eccentric remodelling.

ventricular systole, a conduit during early diastole and contracting during late diastole to aid ventricular filling. Echocardiography is validated for the assessment of LA structure. M-mode echocardiography technique may evaluate the LA size by assessing the anteroposterior linear LA diameter (LAD), a widely used method in clinical practice and research. ${ }^{12}$

Similarly to other cardiac structures, LA scales with body size. ${ }^{1}{ }^{3}$ Several indexing methods have been proposed to adjust LA size for anthropometrics, but indexing by body surface area (BSA) is the most common and recommended. ${ }^{1}$ Analyses of indexing left ventricular structure for body size indicate that BSA may over adjust for obesity-related increases in the left ventricular mass. ${ }^{4}$ However, indexing cardiac structures to height theoretically reduces confounding effects and improves clinical management when compared with BSA. ${ }^{5}$

The National Heart, Lung and Blood Institute's Coronary Artery Risk Development in Young Adults (CARDIA) study investigates prospectively $\mathrm{CV}$ disease risk factors and 
subclinical disease in a young population. In the CARDIA study, LAD has an association with subclinical atherosclerosis independent of other coronary artery disease risk factors. ${ }^{6}$ There is no information on longitudinal determinants of LAD during the transition from young adulthood to middle age.

This study assesses how modifiable risk factors in young adulthood associate with LA size over a 20-year period. We investigate how early adult and 20-year changes in modifiable $\mathrm{CV}$ factors predict $\mathrm{LAD}$ at age $43-55$ years. In addition, we explore how this prediction is affected by indexing LAD by BSA or height.

\section{METHODS}

\section{Study design and sample}

The CARDIA study is a prospective observational investigation that has completed 25 years of follow-up. ${ }^{7}$ Between 1985 and 1986, 5115 African-American and white participants (aged 18-30 years) were enrolled in four field centres (Birmingham, Alabama; Oakland, California; Chicago, Illinois; and Minneapolis, Minnesota, USA). Then, the participants underwent follow-up examination in years $0,2,5,7,10,15,20$ and 25 , with echocardiograms performed in the entire cohort at years 5 and 25. We included participants who underwent echocardiography assessment and had data on LAD and modifiable $\mathrm{CV}$ risk factors at CARDIA examination years 5 and 25. From the 3240 participants who attended CARDIA examination year 5 (baseline in this study) and examination year-25, 24 participants did not have echocardiography performed at year 25 and 313 had incomplete data on covariates at CARDIA examination year 5 or 25 . The final analytic cohort for this study included 2903 participants. All participants gave written informed consent.

\section{Echocardiography}

The CARDIA year 5 echocardiography standard protocol has been described. ${ }^{8}$ Briefly, echocardiograms were performed in each field centre using an Acuson cardiac ultrasound machine (Siemens Healthcare; Erlangen, Germany), recorded in super-VHS tapes, and then interpreted following the American Society of Echocardiography (ASE) recommendations ${ }^{9}$ at a single reading centre (University of California, Irvine, USA). In the field centres, parasternal long-axis two-dimensional views were used to guide the assessment of M-mode anteroposterior images from the aortic root and the left atrium. During the echocardiography interpretation, the LA linear dimension was measured from the leading edge of the posterior aortic wall to the leading edge of the posterior LA wall. CARDIA examination year 25 echocardiography used Artida cardiac ultrasound machines (Toshiba Medical Systems, Otawara, Japan), following acquisition and interpretation protocols similar to examination year 5. LADs from examination years 5 and 25 were indexed to BSA and height (in meters) from the corresponding examination year.
Risk factors assessment

We explored biological and lifestyle risk factors by assessing the association between modifiable risk factors and LA size. Although other factors may be related to LA size, modifiable risk factors were chosen among major $\mathrm{CV}$ risk factors known to be associated to LAD and that could be favourably modified by a healthy lifestyle, such as blood pressure (BP), use of medication for hypertension, body mass index (BMI), heart rate (HR), smoking status, alcohol use, physical activity score and diabetes status.

Assessment methods for risk factor variables have been described for the CARDIA study. ${ }^{10}$ Briefly, use of medication, alcohol consumption (in milliliter of ethanol consumed per day) and smoking status (not smoking or current smoker) were assessed using questionnaires. After 5 min rest, the last two of a total of three measurements of $\mathrm{BP}$ were averaged for computing systolic (SBP) and diastolic BP (DBP) values; and HR was assessed in $30 \mathrm{~s}$. A physical activity score was obtained from the CARDIA Physical Activity History, as previously described. ${ }^{5}$ In the CARDIA study, the presence of diabetes was assessed at each examination based on a combination of history of medication use (every visit), fasting glucose $\geq 126 \mathrm{mg} / \mathrm{dL}$ (years $0,7,10,15,20$ and 25), glucose tolerance test (years 10,20 and 25 ; glucose $\geq 200 \mathrm{mg} / \mathrm{dL}$ ) or glycated haemoglobin $\geq 6.5 \%$ (years 20 and 25). We defined presence of diabetes at baseline if any of these criteria was present at examination year 5 . New cases of diabetes at examination year 25 were computed if the criteria for diabetes were established over the period between examination year 5 and the end of follow-up at examination year 25.

\section{Data analysis}

Continuous variables were described as mean $\pm \mathrm{SD}$ and categorical variables in per cents. For each participant, we compared all parameters assessed at CARDIA examination years 5 and 25. The differences between mean values were tested by paired t test and between proportions by McNemar's.

Cross-sectional relations between risk factors and LA size (both at year 5) were assessed by multivariate linear regression. The longitudinal relation between LA size at years 5 and 25 was also assessed. Multivariable regression models assessed the influence of examination year 5 modifiable risk factors on examination year $25 \mathrm{LAD}$, $\mathrm{LAD} / \mathrm{BSA}$ and $\mathrm{LAD} /$ height. In sequence, multivariable regression models assessed the influence of modifiable risk factors at examination year 5 and their 20-year change on examination year $25 \mathrm{LAD}, \mathrm{LAD} / \mathrm{BSA}$ and $\mathrm{LAD} /$ height. Ethnic-specific analysis for $\mathrm{LAD} /$ height was also performed for the fully adjusted model to explore ethnic particularities (for results see online supplementary material).

The association between LAD and BP was explored by including antihypertensive medication use with SBP or DBP as covariates in the regression models. The relation between diabetes and LAD was assessed using presence of diabetes at baseline and new cases of diabetes at 
examination year 25. All multivariable regression models were adjusted for other known CV risk factors, here defined as non-modifiable by a healthy lifestyle: age, ethnicity, gender and examination year 5 LA size. Maximum education attained was tested, but did not show an association with LAD, and, therefore, was not included in the regression models (data not shown).

\section{RESULTS}

The participant characteristics at CARDIA examination years 5 and 25 are shown in table 1 . Over 20 years (CARDIA examination 5-25), alcohol consumption, BMI and SBP increased significantly in the study cohort, while HR, tobacco use and the physical activity score decreased significantly. Although statistically significant, changes in mean alcohol consumption, heart rate and cigarette use over 20 years were not substantial. In the same period, the proportion of participants with hypertension and diabetes increased. $\mathrm{LAD}$ and $\mathrm{LAD} /$ height were modestly higher over the follow-up period, but LAD/BSA had a slight decrease.

In a cross sectional analysis at CARDIA examination year-5, BMI was directly related to $\mathrm{LAD}$ and $\mathrm{LAD} /$ height and an inverse relation was found for LAD/BSA. Being a current smoker and having higher resting HR were consistently related to higher $\mathrm{LAD}, \mathrm{LAD} / \mathrm{BSA}$ and LAD/height. Antihypertensive medication use, SBP and physical activity had significant direct relations to LAD, but no association was found after indexing LAD by height or BSA. Neither alcohol use nor presence of diabetes had cross-sectional association with LA size (table 2).

Table 3 shows the multivariable linear regression models for the influence of examination year 5 modifiable $\mathrm{CV}$ risk factors on $\mathrm{LAD}$ and its indices over a 20-year follow-up period, adjusted for age, race, gender and baseline LA size. Higher values of baseline SBP were significantly related to higher $\mathrm{LAD}$ and $\mathrm{LAD} / \mathrm{BSA}$ over 20 years, with marginal significance for LAD/ height. No significant relationship between LA size and DBP was found, when DBP was tested replacing SBP in the model. Higher BMI was related to higher CARDIA examination year $25 \mathrm{LAD}$ and $\mathrm{LAD} /$ height. However, the opposite direction was found for baseline BMI in the regression model for $\mathrm{LAD} / \mathrm{BSA}$. Increase in baseline HR was related to lower values of LAD and LAD indices. Neither baseline smoking status, alcohol consumption nor physical activity score had significant prediction ability for LAD or LAD indices. The presence of diabetes at baseline was associated with enlarged LA size after a 20-year follow-up period.

In table 4, we show the results for multivariable regression models assessing simultaneously baseline (CARDIA examination year 5) and 20-year change covariates for the endpoints (measured at CARDIA examination year 25) of $\mathrm{LAD}, \mathrm{LAD} / \mathrm{BSA}$ and $\mathrm{LAD} /$ height. Lower baseline HR and HR 20-year changes showed a significant relationship to higher LAD and LAD indices at CARDIA examination year 25. Higher values of baseline SBP and SBP 20-year changes also related directly to LAD and LAD indices at year 25. Compared with SBP, DBP had a weaker relation to LA size when tested in the same models (see online supplementary table S1). Higher $\mathrm{BMI}$ and BMI changes were related to enlargement in $\mathrm{LAD}$ and $\mathrm{LAD} /$ height. However, again an inverse correlation was found for baseline BMI and changes when LAD/BSA was used as the endpoint. Neither smoking status, alcohol consumption nor physical activity score at baseline or over 20-year changes had significant

Table 1 Participant characteristics at examination year 5 and after a 20-year follow-up period $(n=2903)$

\begin{tabular}{|c|c|c|c|}
\hline Variables & $\begin{array}{l}\text { Examination year } 5 \\
\text { Mean (SD) }\end{array}$ & $\begin{array}{l}\text { Examination year } 25 \\
\text { Mean (SD) }\end{array}$ & p Value \\
\hline Age (years) & $30(4)$ & $50(4)$ & NA \\
\hline BMI $\left(\mathrm{kg} / \mathrm{m}^{2}\right)$ & $26(6)$ & $30(7)$ & $<0.0001$ \\
\hline $\mathrm{SBP}(\mathrm{mm} \mathrm{Hg})$ & $107(11)$ & $119(16)$ & $<0.0001$ \\
\hline HR (bpm) & $68(10)$ & $66(10)$ & $<0.0001$ \\
\hline Alcohol use (mL/day) & $11(22)$ & $12(23)$ & 0.006 \\
\hline Physical activity score (units) & $378(289)$ & $339(272)$ & $<0.0001$ \\
\hline Cigarettes (number/day) & $3(7)$ & $2(5)$ & $<0.0001$ \\
\hline LAD (cm) & $35(5)$ & $37(5)$ & $<0.0001$ \\
\hline $\mathrm{LAD} / \mathrm{BSA}\left(\mathrm{cm} / \mathrm{m}^{2}\right)$ & $1.9(0.2)$ & $1.8(0.2)$ & $<0.0001$ \\
\hline \multirow[t]{2}{*}{ LAD/height $(\mathrm{cm} / \mathrm{m})$} & $2.1(0.3)$ & $2.2(0.3)$ & $<0.0001$ \\
\hline & Proportion & Proportion & \\
\hline White ethnicity & $56 \%$ & NA & NA \\
\hline Male gender & $44 \%$ & NA & NA \\
\hline Current smoker & $26 \%$ & $16 \%$ & $<0.0001$ \\
\hline Hypertension & $4 \%$ & $34 \%$ & $<0.0001$ \\
\hline Diabetes & $1 \%$ & $14 \%$ & 0.0001 \\
\hline
\end{tabular}

$\mathrm{p}$ Values for differences between mean values were tested by paired t test and between proportions by McNemar's test. BMI, body mass index; BSA, body surface area; HR, heart rate; LAD, left atrial diameter assessed by M-mode echocardiography; NA, not applicable; SBP, systolic blood pressure. 
Table 2 Multivariable linear regression for cross-sectional association of modifiable risk factors with left atrial size, both measured at examination year $5(n=2903)$

\begin{tabular}{|c|c|c|c|c|c|c|}
\hline \multirow[b]{2}{*}{ Variable } & \multicolumn{2}{|c|}{$\begin{array}{l}\text { Unindexed LAD (cm) } \\
\left(R^{2}=0.29\right)\end{array}$} & \multicolumn{2}{|c|}{$\begin{array}{l}\text { LAD/BSA }\left(\mathrm{cm} / \mathrm{m}^{2}\right) \\
\left(\mathrm{R}^{2}=0.08\right)\end{array}$} & \multicolumn{2}{|c|}{$\begin{array}{l}\text { LAD/height }(\mathrm{cm} / \mathrm{m}) \\
\left(R^{2}=0.25\right)\end{array}$} \\
\hline & Coefficient & p Value & Coefficient & p Value & Coefficient & p Value \\
\hline Presence of diabetes & -0.065 & 0.507 & -0.028 & 0.626 & -0.034 & 0.561 \\
\hline BMI $\left(5 \mathrm{~kg} / \mathrm{m}^{2}\right)$ & 0.183 & $<0.0001$ & -0.025 & $<.0001$ & 0.113 & $<0.0001$ \\
\hline SBP $(10 \mathrm{~mm} \mathrm{Hg})$ & 0.021 & 0.005 & 0.004 & 0.374 & 0.007 & 0.125 \\
\hline $\mathrm{HR}$ (10 bpm) & -0.066 & $<0.0001$ & -0.033 & $<.0001$ & -0.037 & $<0.0001$ \\
\hline Current smoker & 0.070 & $<0.0001$ & 0.041 & $<.0001$ & 0.043 & $<0.0001$ \\
\hline Using medication for HTN & 0.174 & 0.005 & 0.062 & 0.0848 & 0.097 & 0.009 \\
\hline Alcohol consumption (20 mL/day) & 0.010 & 0.159 & 0.007 & 0.0714 & 0.008 & 0.058 \\
\hline Physical activity score (300 u) & 0.026 & 0.001 & 0.006 & 0.2091 & 0.009 & 0.049 \\
\hline
\end{tabular}

Cross-sectional regression models adjusted for age, ethnicity and gender.

BMI, body mass index; BSA, body surface area; HR, heart rate; HTN, hypertension; LAD, left atrial dimension; SBP, systolic blood pressure.

prediction ability for LAD or LAD indices. Higher LAD was associated with baseline diabetes, but no statistical significance was found for the presence of diabetes at examination year 25. Caucasian and African-American participants showed similar results for the influence of risk factors on LA size (see online supplementary table $\mathrm{S} 2$ ).

\section{DISCUSSION}

In this study, we show how modifiable CV risk factors in a large cohort of young adults are associated with LAD over a 20-year period. During early adulthood, the most robust predictors for LA enlargement over a 20-year follow-up period were higher SBP, lower heart rate and higher BMI. In addition, the presence of diabetes at baseline showed a significant relation to high LA size at examination year 25. However, alcohol use, physical activity and smoking status did not show significant longitudinal influence.

Cardiac remodelling plays a central role in CV disease and may be characterised by heart chamber enlargement and dysfunction. The LA remodelling process strongly relates to increase in left ventricular filling pressures. Furthermore, LA structure and function show important associations to $\mathrm{CV}$ risk burden and clinical events prediction. ${ }^{2} 411$ In a longitudinal assessment over 10 years in the CARDIA cohort, LAD assessed on 2724 participants at examination year 5 was associated with the presence of coronary calcium at CARDIA examination year 15 (2000-2001), independent of other risk factors such as age, sex, race, BMI, SBP, smoking and lipids. ${ }^{6}$ The intensity of exposure to $\mathrm{CV}$ risk factors in youth correlates to early coronary disease. ${ }^{12}$ However, there are limited data regarding how long-term risk factor exposure influences LA size.

LAD assessment by M-mode echocardiography is a practical, low cost and validated method. ${ }^{9} 1314$ It has high consistency, but may lack accuracy as it is based on the relationship between anteroposterior LAD and other spatial dimensions in the LA remodelling process. ${ }^{1}$ Despite this intrinsic limitation, previous studies have shown the association between increased LAD by M-mode echocardiography and incident CV outcomes, particularly atrial fibrillation and cerebrovascular events. $^{15} 16$ Moreover, the LIFE Study followed with

Table 3 Multivariable linear regression for association of examination year 5 (baseline) modifiable risk factors with left atrial size over a 20-year follow-up period $(n=2903)$

\begin{tabular}{|c|c|c|c|c|c|c|}
\hline \multirow[b]{2}{*}{ Variable } & \multicolumn{2}{|c|}{$\begin{array}{l}\text { Unindexed LAD (cm) } \\
\left(R^{2}=0.29\right)\end{array}$} & \multicolumn{2}{|c|}{$\begin{array}{l}\text { LAD/BSA }\left(\mathrm{cm} / \mathrm{m}^{2}\right) \\
\left(\mathrm{R}^{2}=0.22\right)\end{array}$} & \multicolumn{2}{|c|}{$\begin{array}{l}\text { LAD/height }(\mathrm{cm} / \mathrm{m}) \\
\left(R^{2}=0.26\right)\end{array}$} \\
\hline & Coefficient & p Value & Coefficient & p Value & Coefficient & p Value \\
\hline Diabetes at baseline & 0.231 & 0.029 & 0.119 & 0.030 & 0.142 & 0.024 \\
\hline BMI $\left(5 \mathrm{~kg} / \mathrm{m}^{2}\right)$ & 0.108 & $<0.0001$ & -0.037 & $<0.0001$ & 0.065 & $<0.0001$ \\
\hline SBP $(10 \mathrm{~mm} \mathrm{Hg})$ & 0.020 & 0.011 & 0.010 & 0.016 & 0.009 & 0.061 \\
\hline HR (10 bpm) & -0.019 & 0.029 & -0.005 & 0.257 & -0.010 & 0.042 \\
\hline Current smoker & 0.018 & 0.350 & 0.005 & 0.583 & 0.015 & 0.181 \\
\hline Using medication for HTN & -0.041 & 0.539 & 0.011 & 0.741 & -0.024 & 0.549 \\
\hline Alcohol consumption (20 mL/day) & -0.010 & 0.188 & 0.004 & 0.319 & -0.004 & 0.370 \\
\hline Physical activity score (300 u) & 0.005 & 0.564 & 0.004 & 0.356 & -0.001 & 0.846 \\
\hline
\end{tabular}

Models adjusted for age at baseline, ethnicity, gender and left atrial size at baseline. Left atrial size at baseline refers to unindexed LAD, LAD/ $\mathrm{BSA}$ or LAD/height, according to the endpoint in the regression model.

BMI, body-mass index; BSA, body surface area; HR, heart rate; HTN, hypertension; LAD, left atrial dimension; SBP, systolic blood pressure. 
Table 4 Multivariable linear regression for influence of CARDIA examination year 5 (baseline) and 20-year change modifiable risk factors on left atrial size at CARDIA examination year $25(n=2903)$

\begin{tabular}{|c|c|c|c|c|c|c|}
\hline \multirow[b]{2}{*}{ Variable } & \multicolumn{2}{|c|}{$\begin{array}{l}\text { Unindexed LAD (cm) } \\
\left(R^{2}=0.35\right)\end{array}$} & \multicolumn{2}{|c|}{$\begin{array}{l}\text { LAD/BSA }\left(\mathrm{cm} / \mathrm{m}^{2}\right) \\
\left(\mathrm{R}^{2}=0.31\right)\end{array}$} & \multicolumn{2}{|c|}{$\begin{array}{l}\text { LAD/height }(\mathrm{cm} / \mathrm{m}) \\
\left(\mathrm{R}^{2}=0.33\right)\end{array}$} \\
\hline & Coefficient & p Value & Coefficient & p Value & Coefficient & p Value \\
\hline Diabetes at baseline (year 5) & 0.254 & 0.013 & 0.122 & 0.019 & 0.154 & 0.011 \\
\hline Diabetes at follow-up, but not baseline & 0.017 & 0.480 & -0.001 & 0.963 & 0.006 & 0.677 \\
\hline BMI at baseline $\left(5 \mathrm{~kg} / \mathrm{m}^{2}\right)$ & 0.108 & $<0.0001$ & -0.040 & $<0.0001$ & 0.065 & $<0.0001$ \\
\hline BMI changes $\left(5 \mathrm{~kg} / \mathrm{m}^{2}\right)$ & 0.126 & $<0.0001$ & -0.076 & $<0.0001$ & 0.075 & $<0.0001$ \\
\hline SBP at baseline $(10 \mathrm{~mm} \mathrm{Hg})$ & 0.034 & $<0.0001$ & 0.014 & 0.002 & 0.018 & $<0.001$ \\
\hline SBP changes $(10 \mathrm{~mm} \mathrm{Hg})$ & 0.025 & $<0.0001$ & 0.012 & $<0.0001$ & 0.017 & $<0.0001$ \\
\hline $\mathrm{HR}$ at baseline ( 5 beats/30 s) & -0.048 & $<0.0001$ & -0.021 & $<0.0001$ & -0.028 & $<0.0001$ \\
\hline HR changes ( 5 beats $/ 30 \mathrm{~s})$ & -0.049 & $<0.0001$ & -0.025 & $<0.0001$ & -0.030 & $<0.0001$ \\
\hline \multicolumn{7}{|l|}{ Smoking status (vs never smoked) } \\
\hline Not baseline, yes Y25 & -0.057 & 0.295 & -0.037 & 0.185 & -0.036 & 0.264 \\
\hline Yes baseline, no Y25 & 0.007 & 0.771 & -0.003 & 0.815 & 0.004 & 0.796 \\
\hline Yes baseline, yes Y25 & 0.010 & 0.680 & 0.010 & 0.381 & 0.013 & 0.325 \\
\hline \multicolumn{7}{|l|}{ Medication for HTN (vs never used) } \\
\hline Not baseline, yes Y25 & 0.031 & 0.118 & 0.020 & 0.044 & 0.025 & 0.035 \\
\hline Yes baseline, no Y25 & 0.055 & 0.685 & 0.008 & 0.904 & 0.024 & 0.769 \\
\hline Yes baseline, yes Y25 & -0.012 & 0.865 & 0.018 & 0.633 & 0.002 & 0.970 \\
\hline Alcohol consumption at baseline (20 mL/day) & -0.002 & 0.850 & 0.005 & 0.301 & 0.001 & 0.799 \\
\hline Alcohol consumption changes ( $20 \mathrm{~mL} /$ day) & 0.006 & 0.423 & 0.006 & 0.128 & 0.004 & 0.322 \\
\hline Physical activity score at baseline $(300 \mathrm{u})$ & 0.013 & 0.217 & 0.003 & 0.574 & 0.004 & 0.541 \\
\hline Physical activity score changes (300 u) & 0.008 & 0.402 & 0.004 & 0.383 & 0.005 & 0.399 \\
\hline
\end{tabular}

echocardiograms 939 hypertensive patients for 4.8 years and found that enlarged baseline LAD increased risk for atrial fibrillation whereas reduction of LAD reduced the risk in models adjusted for age, LV mass, SBP and Framingham risk score. ${ }^{17}$

Similar to our study, cross-sectional relations between $\mathrm{CV}$ risk factors and LAD have been reported in the literature. Cuspidi et $a l^{18}$ found significantly higher BMI and SBP with enlarged LAD in a population of 2500 uncomplicated patients with hypertension. Tsang et $a l^{15}$ investigated 423 patients and also found cross-sectional relations of LAD with BMI and SBP. In a cross-sectional assessment of 4059 CARDIA participants at examination year 5 , a low $\mathrm{CV}$ risk burden was associated with more favourable values for LA size. In this study, Gidding et $a l^{19}$ showed that higher BMI, higher SBP, lower heart rate, tobacco use, higher serum glucose and higher selfreported physical activity were independently associated with enlarged $\mathrm{LAD} /$ height.

There are limited data on the longitudinal determinants of LA size. In this study, we assessed a generally healthy cohort examined at ages $23-35$ and $43-55$ years. $\mathrm{BP}$, particularly SBP and BMI emerged as very robust risk factors associated with $\mathrm{LAD}$ and $\mathrm{LAD}$ change over 20 years. McManus $e t a l^{20}$ reported similar findings following an older population of 4403 Framingham Study participants over a 16-year follow-up period. BP and BMI were the major factors related to LAD enlargement. BP and obesity are known determinants of $\mathrm{LV}$ diastolic dysfunction and cardiac remodelling, ${ }^{21} 22$ strongly associated with elevated filling pressures and LA enlargement. In fact, higher BP was a consistent determinant of LAD enlargement in our study. In our cohort of young participants, the association between BP and LA size was weaker in cross-sectional as compared with longitudinal regression models. This emphasises the importance of chronic exposure to high $\mathrm{BP}$ and subclinical cardiac endpoints, including LA size.

Obesity, as assessed by BMI, strongly relates to CV risk. ${ }^{23} \mathrm{BMI}$ was also consistently related to LA size in our study, but the relationship varied depending on the method used to index LAD. In this regard, indexing LAD by BSA may overadjust for deleterious effects of excess adiposity-producing values that underestimate risk in obese participants. ${ }^{24} 25$ This is suggested by our results where the relationship between change in BMI and LAD/BSA was inverse despite the knowledge that BMI is strongly related to LAD in cross-sectional analyses, and BMI and LAD contribute to CVD risk. A crosssectional study of 244 children found an independent association of LAD with body fat mass, body fat as a percentage of body mass, abdominal fat mass and body fat distribution. ${ }^{26}$ Adjusting CV parameters for height alone appear to provide more stable longitudinal assessment. ${ }^{3}$

In our study, resting HR was associated to LAD and $\mathrm{LAD} /$ height but not to LAD/BSA at CARDIA examination year 25 , when only year 5 variables were included in the regression models (table 2). Furthermore, lower 
$\mathrm{HR}$ at baseline and its 20-year decrease emerged as significant determinants of LAD enlargement over the 20-year follow-up period (table 3). HR is inversely related to stroke volume at rest and a higher resting HR has shown an association with adverse events. ${ }^{27-29}$ However, there may be a threshold effect for the adverse association of elevated HR. Values above $80 \mathrm{bpm}$ may have a stronger association to CV risk in older populations, possibly related to the association of a higher oxygen consumption with higher prevalence of existing coronary disease and adverse cardiac function in this population. ${ }^{30}$ These data suggest that some changes in LA size may be adaptive as opposed to adverse and are consistent with the cross-sectional inverse relationship between $\mathrm{HR}$ and $\mathrm{LAD}$ reported in the literature. $^{15} 1819$

Diabetes is a known risk factor for CV disease including heart failure. However, LA size had no crosssectional association with diabetes in our study. Moreover, LA enlargement was related to baseline diabetes, but not its new development after 20 years. These findings suggest that the period of exposure to diabetes may play an important role in atrial remodelling.

LAD enlargement may be related to an adaptation process in exercise conditioning. Evidence of the association between physical activity and LAD have been reported in high-performance athletes, ${ }^{31}{ }^{32}$ and also in a cross-sectional analysis of CARDIA participants. ${ }^{19}$ However, our study did not find a significant relationship between LAD and physical activity at baseline or with its 20-year changes. This is likely secondary to the small number of elite athletes in the cohort. Smoking is a major CV risk factor and is related to left ventricular fibrosis, mass and diastolic function. In fact, being a smoker had a cross-sectional relation with the higher LA size in our study. However, smoking status was not a significant longitudinal determinant of LA size in young adults over a 20-year follow-up period. Previous crosssectional studies also failed to find significant relations regarding tobacco use and LA size. ${ }^{15}{ }^{18}$ Alcohol use also had no significant relation to LAD. Alcohol consumption has a controversial association to $\mathrm{CV}$ risk, probably influenced by the amount and type of the agent used. ${ }^{33}$ In this regard, our study is limited by not accounting for the type of beverage used by the participants.

In a large biracial cohort of young adults, BP and BMI played a major role in LA enlargement over a 20-year period; resting HR and its 20-year changes were inversely related to LAD. In addition, diabetes at age 23-35 years, but not incident, was significantly related to a higher LAD size. Particularly interesting results were found regarding the $\mathrm{LAD}$ indexing process, with negative correlations between change in $\mathrm{BMI}$ and $\mathrm{LAD} / \mathrm{BSA}$ values. Therefore, LAD indexed to BSA may not be the best indexing method for longitudinal assessment of LA size. Future studies directly comparing indexing methods in clinical event prediction are needed to establish the best method for indexing LA size.
Author affiliations

${ }^{1}$ Department of Cardiology, Johns Hopkins University, Baltimore, Maryland, USA

${ }^{2}$ Department of Internal Medicine/Cardiology, Universidade Federal do Vale do São Francisco, Petrolina, Pernambuco, Brazil

${ }^{3} \mathrm{~A}$ I DuPont Hospital for Children, Wilmington, Delaware, USA

${ }^{4}$ Northwestern University, Chicago, Illinois, USA

${ }^{5}$ Kaiser Permanente, Oakland, California, USA

${ }^{6}$ University of Minnesota, Minneapolis, Minnesota, USA

${ }^{7}$ University of Alabama at Birmingham, Birmingham, Alabama, USA

${ }^{8}$ Escola Bahiana de Medicina, Salvador, Bahia, Brazil

Acknowledgements The authors thank the other investigators, the staff and the participants of the CARDIA study for their valuable contributions. This manuscript has been reviewed by CARDIA for scientific content.

Contributors All the authors contributed to interpretation of the data, revising the article critically for important intellectual content and to the final approval of the version to be published. Also, JACL and AAA contributed to drafting the article. JACL, AAA, KL and LAC contributed to conception and design of the article, and analysis of the data.

Funding The Coronary Artery Risk Development in Young Adults Study (CARDIA) is conducted and supported by the National Heart, Lung, and Blood Institute in collaboration with the University of Alabama at Birmingham (HHSN268201300025C and HHSN268201300026C), Northwestern University (HHSN268201300027C), University of Minnesota (HHSN268201300028C), Kaiser Foundation Research Institute (HHSN268201300029C) and Johns Hopkins University School of Medicine (HHSN268200900041C). CARDIA is also partially supported by the Intramural Research Programme of the National Institute on Aging.

Competing interests Dr Armstrong was funded by Universidade Federal do Vale do São Francisco (Petrolina, Brazil) and by the Johns Hopkins University (Baltimore, Maryland).

Ethics approval The study was approved by the institutional review boards (IRBs) of the participant institutions. Johns Hopkins IRB and in all CARDIA sites.

Provenance and peer review Not commissioned; externally peer reviewed.

Data sharing statement The CARDIA study has provided the National Heart, Lung, and Blood Institute data repository datasets. Additional information can be found on the CARDIA Study website http://www.cardia.dopm.uab.edu/

Open Access This is an Open Access article distributed in accordance with the Creative Commons Attribution Non Commercial (CC BY-NC 3.0) license, which permits others to distribute, remix, adapt, build upon this work noncommercially, and license their derivative works on different terms, provided the original work is properly cited and the use is non-commercial. See: http:// creativecommons.org/licenses/by-nc/3.0/

\section{REFERENCES}

1. Lang RM, Bierig M, Devereux RB, et al. Recommendations for chamber quantification: a report from the American Society of Echocardiography's Guidelines and Standards Committee and the Chamber Quantification Writing Group, developed in conjunction with the European Association of Echocardiography, a branch of the European Society of Cardiology. J Am Soc Echocardiogr 2005;18:1440-63.

2. Tops LF, van der Wall EE, Schalij MJ, et al. Multi-modality imaging to assess left atrial size, anatomy and function. Heart 2007;93:1461-70

3. Dewey FE, Rosenthal D, Murphy DJ Jr, et al. Does size matter? Clinical applications of scaling cardiac size and function for body size. Circulation 2008;117:2279-87.

4. Edvardsen T. The continued discovery of left atrial function. Eur Heart J Cardiovasc Imaging 2012;13:203-4.

5. Sidney S, Jacobs DR Jr, Haskell WL, et al. Comparison of two methods of assessing physical activity in the Coronary Artery Risk Development in Young Adults (CARDIA) Study. Am J Epidemiol 1991;133:1231-45.

6. Gardin JM, Iribarren C, Detrano RC, et al. Relation of echocardiographic left ventricular mass, geometry and wall stress 
and left atrial dimension to coronary calcium in young adults (the CARDIA study). Am J Cardiol 2005;95:626-9.

7. Friedman GD, Cutter GR, Donahue RP, et al. CARDIA: study design, recruitment, and some characteristics of the examined subjects. J Clin Epidemiol 1988;41:1105-16.

8. Gardin JM, Wagenknecht LE, Anton-Culver H, et al. Relationship of cardiovascular risk factors to echocardiographic left ventricular mass in healthy young black and white adult men and women. The CARDIA study. Coronary Artery Risk Development in Young Adults. Circulation 1995;92:380-7.

9. Sahn DJ, DeMaria A, Kisslo J, et al. Recommendations regarding quantitation in M-mode echocardiography: results of a survey of echocardiographic measurements. Circulation 1978;58:1072-83.

10. Loria CM, Liu K, Lewis CE, et al. Early adult risk factor levels and subsequent coronary artery calcification: the CARDIA study. J Am Coll Cardiol 2007;49:2013-20.

11. Rosca M, Lancellotti P, Popescu BA, et al. Left atrial function: pathophysiology, echocardiographic assessment and clinical applications. Heart 2011;97:1982-9.

12. Celermajer DS, Ayer JG. Childhood risk factors for adult cardiovascular disease and primary prevention in childhood. Heart 2006;92:1701-6.

13. Abhayaratna WP, Seward JB, Appleton CP, et al. Left atrial size: physiologic determinants and clinical applications. J Am Coll Cardiol 2006;47:2357-63.

14. Kizer JR, Bella JN, Palmieri V, et al. Left atrial diameter as an independent predictor of first clinical cardiovascular events in middle-aged and elderly adults: the Strong Heart Study (SHS). Am Heart J 2006;151:412-18.

15. Tsang TS, Abhayaratna WP, Barnes ME, et al. Prediction of cardiovascular outcomes with left atrial size: is volume superior to area or diameter? J Am Coll Cardiol 2006;47:1018-23.

16. Laukkanen JA, Kurl S, Eranen J, et al. Left atrium size and the risk of cardiovascular death in middle-aged men. Arch Intern Med 2005;165:1788-93.

17. Wachtell K, Gerdts E, Aurigemma GP, et al. In-treatment reduced left atrial diameter during antihypertensive treatment is associated with reduced new-onset atrial fibrillation in hypertensive patients with left ventricular hypertrophy: the LIFE study. Blood Press 2010;19:169-75.

18. Cuspidi C, Meani S, Fusi V, et al. Prevalence and correlates of left atrial enlargement in essential hypertension: role of ventricular geometry and the metabolic syndrome: the Evaluation of Target Organ Damage in Hypertension study. J Hypertens 2005; 23:875-82.
19. Gidding SS, Carnethon MR, Daniels S, et al. Low cardiovascular risk is associated with favorable left ventricular mass, left ventricular relative wall thickness, and left atrial size: the CARDIA study. J Am Soc Echocardiogr 2010;23:816-22.

20. McManus DD, Xanthakis V, Sullivan LM, et al. Longitudinal tracking of left atrial diameter over the adult life course: clinical correlates in the community. Circulation 2010;121:667-74.

21. Erdogan D, Caliskan M, Yildirim I, et al. Effects of normal blood pressure, prehypertension and hypertension on left ventricular diastolic function and aortic elastic properties. Blood Press 2007;16:114-21.

22. Gjesdal O, Bluemke DA, Lima JA. Cardiac remodeling at the population level-risk factors, screening, and outcomes. Nat Rev Cardiol 2011;8:673-85.

23. Logue $\mathrm{J}$, Murray HM, Welsh $\mathrm{P}$, et al. Obesity is associated with fatal coronary heart disease independently of traditional risk factors and deprivation. Heart 2011;97:564-8.

24. Armstrong AC, Gjesdal O, Almeida A, et al. Left ventricle mass by cardiac magnetic resonance and echocardiography: the multi-ethnic study of atherosclerosis. Echocardiography 2013; [Epub ahead of print 10 Aug 2013]. doi:10.1111/echo.12303

25. Armstrong AC, Gjesdal O, Wu C, et al. Left ventricular mass assessed by Echocardiography and Cardiac Magnetic Resonance, cardiovascular outcomes, and medical practice. J Am Coll Cardiol Img 2012;5:11.

26. Dencker M, Thorsson O, Karlsson MK, et al. Body fat, abdominal fat and body fat distribution is related to left atrial diameter in young children. Obesity (Silver Spring) 2012;20:1104-8.

27. Palatini P. Elevated heart rate: a "new" cardiovascular risk factor? Prog Cardiovasc Dis 2009;52:1-5.

28. Cooney MT, Vartiainen E, Laatikainen T, et al. Simplifying cardiovascular risk estimation using resting heart rate. Eur Heart $J$ 2010;31:2141-7.

29. Cook S, Togni M, Schaub MC, et al. High heart rate: a cardiovascular risk factor? Eur Heart J 2006;27:2387-93.

30. Perret-Guillaume $C$, Joly $L$, Benetos A. Heart rate as a risk factor for cardiovascular disease. Prog Cardiovasc Dis 2009;52:6-10.

31. Pelliccia A, Maron BJ, Di Paolo FM, et al. Prevalence and clinical significance of left atrial remodeling in competitive athletes. J Am Coll Cardiol 2005;46:690-6.

32. Pelliccia A, Culasso F, Di Paolo FM, et al. Physiologic left ventricular cavity dilatation in elite athletes. Ann Intern Med 1999;130:23-31.

33. Di Castelnuovo A, Costanzo S, di Giuseppe R, et al. Alcohol consumption and cardiovascular risk: mechanisms of action and epidemiologic perspectives. Future Cardiol 2009;5:467-77. 